\title{
MYC overexpression predicts poor prognosis and correlates with immune infiltration in chronic lymphocytic leukemia
}

Dongmei Guo

Taian City Central Hospital

Ping Li

Dezhou People's Hospital

\section{Zan Wang}

Shandong First Medical University

Banban Li

Taian City Central Hospital

Gaofeng Bi

Taian City Central Hospital

Chunpu Li (Dli45li@126.com)

Taian City Central Hospital

\section{Research Article}

Keywords: MYC, chronic lymphocytic leukemia,WGCNA, prognosis, immune cell infiltration

Posted Date: January 24th, 2022

DOI: https://doi.org/10.21203/rs.3.rs-1276445/v1

License: (9) (i) This work is licensed under a Creative Commons Attribution 4.0 International License. Read Full License 


\section{Abstract}

Background: Chronic lymphocytic leukemia (CLL) is a highly genetically heterogeneous disease that represents $30 \%$ of adult leukemia. We aimed to identify survival-associated key genes in CLL and investigate potential immunotherapy targets.

Methods: The survival information and microarray mRNA expression profiles of CLL patients were downloaded from the Gene Expression Omnibus (GEO) and the International Cancer Genome Consortium (ICGC) database. Hub genes were uncovered in CLL. The bioinformatics technology was applied to identify key genes and performe functional analysis. Finally, we explored the role of this key gene and the situation of immune infiltration in the bone marrow.

Results: We uncovered 223 differentially expressed genes (DEGs) as hub genes associated with CLL. MYC was identified as the key gene in CLL. High MYC expression was associated with poor prognosis. The biological pathways such as "Hypoxia", "Myc Targets V2", "P53 pathway" etc, were remarkably linked with MYC high expression phenotype. The expression level of MYC was positively correlated with infiltration levels of Eosinophils and follicular helper T cells, while negatively correlated with infiltration levels of regulatory $T$ cells.

Conclusion: Overexpression of MYC predicts poor prognosis and correlates with immune infiltration. MYC could serve as a potential immunotherapeutic target for CLL.

\section{Introduction}

Chronic lymphocytic leukemia (CLL) is a clonal and proliferative tumor of mature B lymphocytes, characterized by the accumulation of CD5+ and CD19+ B cells in peripheral blood, bone marrow, spleen and lymph nodes [1]. As a highly heterogeneous disease, CLL has significant individual differences in pathogenesis, disease progression, therapeutic effect, and survival period [2]. The disease has an insidious onset and unknown etiology. Recent studies have shown that the tumor microenvironment plays an essential role in the progression of CLL disease. Therefore, how the microenvironment in CLL can promote the survival of leukemia cells deserves further study.

Immune infiltration refers to the abnormal appearance of human immune cells in diseased tissues. The specific mechanism is not completely clear at present. The immune imbalance is an essential part of the pathogenesis of CLL, and there are few studies on the regulation mechanism of immune infiltration in CLL. In recent years, the awareness of immune infiltration has gradually increased. Immune cells infiltrate tumor tissues to constitute the tumor microenvironment. Infiltration of immune cells has been found in various solid tumor tissues, such as lung cancer, breast cancer, colorectal cancer[3-7], and the type of infiltrating immune cells is strongly correlated with the clinical characteristics of these solid tumors, affecting the clinical prognosis of tumor patients [8-9]. Therefore, some researchers have proposed that the infiltration of different immune cells in tumor tissues can be used for tumor risk stratification [10] and becomes a potential drug target to improve patients' survival rate [11]. 
MYC gene is a nucleoprotein proto-oncogene, mainly including c-myc, n-myc, I-myc families. It is involved in the activation of the PTEN/PI3K/AKT pathway, and its encoded product can specifically bind with DNA to promote the opening of cell proliferation-related genes and produce cell proliferation effects [12]. MYC gene plays an essential role in the formation, development and prognosis evaluation of lymphoma [13]. Studies have shown that MYC protein overexpression accounts for $29 \%-47 \%$ in diffuse large B-cell lymphoma (DLBCL) [14-16]. MYC protein overexpression is a poor prognostic factor for DLBCL patients $[12,15]$. Kluk MJ found that a lower overall survival rate in patients with MYC-positive DLBCL treated with $\mathrm{RCHOP}$ regimen[16]. This research uses bioinformatics tools to obtain genes that are most closely related to the occurrence and development of CLL, analyze the immune infiltration of bone marrow, andexplore the role of immune infiltration and immune regulation in the development of the disease. This study can provide the theoretical basis for targeted therapy of CLL [16-17].

\section{Results}

\section{DEG screening and co-expression analysis}

To screen the DEGs between CLL and normal B cells, we carried out differentially expression analysis in GSE31048.Figure 1A showed the volcano plot of the differential mRNA expression analysis. Finally, a total of 396 DEGs were found. Using the "WGCNA" R package, hub modules and relevant genes were identified among these genes. First, the adjacency matrix was converted into a topological overlap matrix (TOM), hence clustering all genes into different modules. In GSE31048, a soft-thresholding power was set at 3 (scale-free $R^{2}=0.90$ ), cut height as 0.25 , and minimal module size as 50 . As a result, the brown module, with the closest association with CLL formation, was identified (Figure 1B).

\section{Identification of hub genes in CLL and the enrichment of these genes}

As shown in the Venn diagram, 223 intersecting genes of the brown module and DEGs were considered hub genes (Figure 2A). To investigate their biological roles, we carried out two types of enrichment assessments: (a) Gene Ontology(GO)enrichment assessment and (b) Kyoto Encyclopedia of Genes and Genomes(KEGG) enrichment assessment. Figure 2B indicates the top 10 enrichment findings in $\mathrm{GO}$. The hub genes under biological processes (BP) terms were mostly linked with leukocyte cell-cell adhesion, $B$ cell activation, regulation of leukocyte cell-cell adhesion, $T$ cell activation, and cell-cell adhesion regulation. As for KEGG pathway enrichment, the hub genes were enriched in Hematopoietic cell lineage, PI3K-Akt signaling pathway, Acute myeloid leukemia, Th17 cell differentiation and Transcriptional misregulation in cancer (Figure 2C).

\section{Determining of key genes in the PPI network}

We imported the 223 hub genes linked to CLL into the STRING web resource to construct aprotein protein interaction (PPI) network. After filtering the PPI complex, 78 nodes and 93 pairs of PPI associations were obtained (Figure 2D). The data were then uploaded into Cytoscape 3.7.2 to compute the network and each node's topological features. According to the three algorithms in the cytoHubba plug-in, we selected 
the top 10 hub genes graded by each algorithm in the PPI network. These genes were located at the central position, serving an essential function in the disease's onset and progression. Seven genes (IL6, CTLA4, CD38, GNB4, SMAD3, PRKACB, and MYC) were linked to CLL (Figure 3A). Further survival analyses were employed to evaluate their effects on the survival of CLL. Briefly, only one gene, MYC, was related to the prognosis of patients. Kaplan-Meier survival analysis discovered that CLL patients with high MYC expression had a shorter overall survival based on the best cutoff 2.24 (Figure 3B). This cutoff value was employed in subsequent subgroup analyses.

\section{MYC -related signaling pathways based on GSEA and GSVA}

We performed Gene Set Enrichment Analysis (GSEA) analysis to investigate the potential mechanism of MYC in CLL prognosis. As shown in Figure 4, genes in the high expression group were enriched in biological pathways, including "Hypoxia", "MYC Targets V2", "TNFA signaling via NFKB", "mTORC1 signaling", "G2Mcheckpoint" and "P53 pathway" (Figure 4). Gene set variation analysis (GSVA) confirmed that they were also significantly up-regulated in the high expression group, further suggesting their importance in CLL progression (Figure 5).

\section{Immune cell infiltration analysis}

To explore the function of MYC in CLL, we set 298 samples in International Cancer Genome Consortium (ICGC). Firstly, due to the significance of immune cells in cancer formation, we evaluated the situation of immune infiltration in bone marrow between low and high MYC groups using CIBERSORT (Figure 6A). Bone marrow with high MYC expression had an elevated percentage of follicular helper T cells, Monocytes, resting dendritic cells, activated Mast cells and Eosinophils, and a lower percentage of naive $B$ cells, regulatory T cells, resting natural killer (NK) cells, M0 Macrophages, M2 Macrophages and resting Mast cells (Figure 6B). Then we explored the association between the expression levels of MYC and immune cells. MYC's expression level was moderate positively correlated with invading levels of eosinophils and follicular helper T cells, while moderate negatively correlated with invading levels of regulatory $T$ cells (Figure 6C-6E).

\section{Discussion}

CLL has significant clinical prognostic heterogeneity. The early parameters used to evaluate the prognosis of CLL mainly includedisease stage (Rai/Binet stage), peripheral blood lymphocyte count, lymphocyte doubling time, bone marrow infiltration pattern, serum $\beta 2$-microspheres Protein ( $\beta 2-M G)$, lactate dehydrogenase (LDH), etc. With the advancesin immunology, cellular/molecular genetics, and molecular biology, the research on CLL prognostic factors has progressed rapidly. Studies have shown that patients with del (17p13) genetic abnormality, immunoglobulin heavy chain variable region (IGVH) mutation status alternative marker ZAP-70 high expression and Binet stage Chave a short survival period. They are essential factors affecting the prognosis of CLL [25]. Besides, many studies have shown that mutations of TP53 (tumorproteinp53) gene and genetic abnormalities such as chromosome 13q deletion (13q-), 11q deletion (11q-) and 17p deletion (17p-) are closely related to the prognosis of CLL [26-27]. With 
the development of next-generation sequencing and gene chip technologies, we have a profound understanding of molecular mechanisms, so it is vital to explore new biological markers that closely link disease progression and clinical prognosis [28].

We used STRING web resource to construct a PPI network and screened seven key genes related to CLL, such as IL6, CTLA4, CD38, GNB4, SMAD3, PRKACB and MYC. Among them, only MYC was related to the prognosis of patients. Survival analysis indicated that CLL patients with high MYC expression had a shorter overall survival. MYC is a proto-oncogene that is abnormally activated in hematological tumors and solid tumors, leading to cell proliferation and growth [29]. It plays a key role in regulating critical biological processes such as the proliferation, differentiation, and apoptosis of hematopoietic cells and malignant leukemia cells, suggesting that it participates in many processes such as the occurrence, development, and evolution of leukemia. The oncogene C-MYC is the most important member of the MYC family. As a potential target for tumor therapy, C-MYC is abnormally highly expressed in a variety of human solid tumors and hematological neoplastic disease [30]. Tumors with high C-MYC expression are characterized by a high proliferation rate, aggressive phenotype, drug resistance, and poor prognosis. CMYC protein has a high expression level in most patients with malignant tumor diseases in hematological tumors, and the prognosis is poor [31]. The abnormal increased proliferation and differentiation disorder of hematopoietic stem/progenitor cells caused by overexpression may be a significant cause of most leukemias [32-33]. Current studies have pointed out that C-MYC can promote the transformation of chronic myeloid leukemia, and its high expression may be one of the mechanisms of disease progression and acute change [34]. In addition, high expression of C-MYC may cause chronic myeloid leukemia cells to reduce imatinib's sensitivity, so the target C-MYC may become a new breakthrough point for the treatment of TKI-resistant chronic myelogenous leukemia [35-36]. The positive expression rate of C-MYC protein, myeloid-derived suppressor cell, and Th17 cell proportion was significantly increased in multiple myeloma patients and was positively correlated with the clinical ISS stage [37].

The GO and KEGG enrichment analysis of hub genes was carried out in this study, suggesting that upregulated genes' biological process is closely related to immune regulation. Leukocyte cell-cell adhesion, B cell activation, regulation of cell-cell adhesion, $T$ cell activation, and regulation of cell-cell adhesion and other biological processes are mainly involved, which further illustrates that CLL is an immune process involving a variety of immune cells, cytokines and complex signal transduction. We performed GSEA and GSVA analyses to explore the potential mechanism of MYC in CLL prognosis. MYC-related signaling pathways are up-regulated, indicating their importance in CLL progression.

Immune infiltration in the tumor microenvironment plays a key role in tumor occurrence and development and affects cancer patients' clinical prognosis [11]. In the development of human solid tumors, immune cells exist in various forms, ranging from infiltrating to overt inflammation. Lymphocytes are usually the largest component of the immune infiltrating site, so they are called "tumor infiltrating lymphocytes (TILs) ". TILs mainly include T lymphocytes, B lymphocytes, NK cells and other immune cells. The phenotypes of these immune lymphocytes can promote or inhibit tumor occurrence and development, 
which is of great significance in the prognosis of tumors. Studies have found that B lymphocytes in TILs are essential biomarkers for tumor treatment and prognosis [38-39], and invasive NK cells have synergistic anti-tumor effects [40]. A retrospective study confirmed that M2 macrophage infiltration was significantly associated with poor breast cancer prognosis [41].

The CLL tumor microenvironment contains a variety of cells such as T lymphocytes, dendritic cells, stromal cells, endothelial cells, etc [42]. Together with lymphocytes and their progenitor cells, these cells form " pseudofollicular " cells, which are distributed in the patient's bone marrow, spleen, and lymph nodes, etc. To further explore the role of MYC and immune cells in CLL, we used the CIBERSORT deconvolution method to assess immune cell subtype distribution. Analysis of the infiltration matrix of immune cells showed that the proportion of follicular helper T cells, Monocytes, resting dendritic cells, activated Mast cells and Eosinophilsin bone marrow with high MYC group increased comparing with low MYC group, while the number of naive B cells, regulatory T cells, resting NK cells, M0 Macrophages, M2 Macrophages and resting Mast cells was reduced. Moreover, correlation analysis between immune cells indicated that the eosinophils and follicular helper T cellswere positively correlated with high MYC expression, and the regulatory $\mathrm{T}$ cells werenegatively correlated.

\section{Conclusion}

In summary, bioinformatics analysis revealed that MYC was highly expressed in CLL, suggesting that it plays a role as an oncogene in CLL. Further analysis demonstrated that MYC overexpression was a useful predictor of poor prognosis of CLL and a molecular marker for prognosis of CLL, suggesting that MYC could be a potential diagnostic and therapeutic target. Another important aspect of this study is that MYC expression is related to immune infiltration. MYC may play a role by regulating the infiltration of immune cells in the tumor microenvironment to provide an effective basis for precise clinical immunotherapy.

\section{Materials And Methods}

\section{Patients and Datasets}

GSE31048, including the RNA sequencing (RNA-seq) data of 188 CLL samples and 33 normal B cell samples, was downloaded from the GEO web resource (http://www.ncbi.nlm.nih.gov/geo). The RNA-seq data and corresponding clinical information of 298 CLL samples were obtained from the ICGC database (https://dcc.icgc.org/projects/CLLE-ES). The raw data from GSE31048 were abstracted and processed to normalize in R software (ver. 3.6.0). The limma R tool was employed to identify DEGs [18]. We uncovered the DEGs with a $\mid \log 2$ fold change $(\mathrm{FC}) \mid>1$ and adjusted $p$-value $<0.05$.

\section{Construction of co-expression network}

The R package was used to filter the genes based on gene expression and variance (standard deviation $\leq 0.5$ ), and 3716 genes were screened out from all genes in GSE31048. Sample clustering was carried out 
to plot the sample tree and uncover the outliers. After that, Pearson's correlation analyses were conducted for pair-wise genes and determined the soft thresholding power $\beta$ value with the pickSoftThreshold tool of the weighted correlation network analysis (WGCNA) [19].

\section{Hub genes identification}

Firstly, the hub modules were defined as the module with the absolute value of the Pearson's correlation of module membership being $>0.8$ and $p$-value $<0.05$. Moreover, the DEGs in hub modules were defined as hub genes.

\section{Enrichment analysis}

We utilized the clusterProfiler package in $\mathrm{R}$ to accomplish functional enrichment analysis consisting of GO and KEGG pathway assessments[20]. GO enrichment constitutes molecular functions, cell components, and BP. P-value of < 0.05 served as the threshold criteria to determine the significant GO terms, as well as KEGG cascades, which were visualized using the bubble diagram or "GOplot" in the R package.

\section{Development of Protein-Protein Interaction (PPI) network}

We imported the genes linked to the clinical progression into the STRING online web platform (https://string-db.org). Then, a PPI network was established with a minimum level of confidence> 0.9 [21]. The plug-in cytoHubba in Cytoscape v3.6.1 was employed to determine the top 10 genes in the network as the core genes via 3 algorithms: Betweenness, Maximal Clique Centrality, and Degree. We intersected the genes obtained by various algorithms to obtain the key genes.

\section{Gene set enrichment analysis and Gene set variation analysis}

GSEA is a computational method for exploring whether an a priori defined set of genes shows statistically significant, concordant differences between two biological states. It has also been applied to analyze the slight changes in the expressions of genes that belong to a key pathway. GSEA was carried out to analyze significant survival differences between MGLL high and low groups on GSEA software [22]. By running GSEA, normalized enrichment scores and nominal $p$-value (NOM $p$-value) were generated for each phenotype's pathways enrichment analysis. Gene sets were considered significantly enriched with NOM $p$-value<0.05, FDR q-value <0.25. Additionally, the GSVA R package was utilized to detect the pathways that are related to each phenotype [23]. An adjusted $P$ value less than 0.05 was considered statistically significant. The reference gene set "h.all.v7.0.symbols" was downloaded from the Molecular Signature Database (http://software.broadinstitute.org/gsea/msigdb/index.jsp).

\section{Assessment of immune cell subtype distribution}

CIBERSORT constitutes a type of deconvolution algorithm for transforming the standardized gene expression matrix into the invading immune cell composition [24]. During the CIBERSORT computation, 
quantification of the enrichment of particular types of cells in complex tissues was performed, and the CIBERSORT findings have been verified by fluorescence-activated cell sorting. We employed LM22 as the reference expression signature set at 1000 permutations. CIBERSORT output of P-value $<0.05$ defined a more accurate prediction of immune cell composition. After that, we selected samples satisfying constraints for further analyses. Herein, 298 samples in ICGC were utilized to estimate the invaded immune cells in the bone marrow. The 22 types of invaded immune cells constituted B cells (memory B cells and naive $B$ cells), neutrophils, $T$ cells (CD8+ T cells, memory resting CD4+ $T$ cells, naive CD4+ $T$ cells, memory activated CD4+ T cells, $\gamma \delta T$ cells, follicular helper $T$ cells, and regulatory $T$ cells), NK cells (activated NK cells and resting NK cells), monocytes, macrophages (M0 macrophages, M2 macrophages, and M1 macrophages), mast cells (activated mast cells and resting mast cells), dendritic cells (resting dendritic cells and activated dendritic cells), and eosinophils. All the explored 22 kinds of immune cell type proportions were to sum up to 1 for each sample.

\section{Abbreviations}

CLL: Chronic lymphocytic leukemia; GEO: Gene Expression Omnibus; ICGC: International Cancer Genome Consortium; DEGs: Differentially expressed genes; DLBCL: Diffuse large B-cell lymphoma;

TOM:Topological overlap matrix; GO: Gene Ontology; KEGG: Kyoto Encyclopedia of Genes and Genomes; PPI: Aprotein protein interaction; GSEA: Gene Set Enrichment Analysis; GSVA: Gene set variation analysis; ICGC: International Cancer Genome Consortium; $\beta 2-M G$ : $\beta 2$-microspheres Protein; LDH: Lactate dehydrogenase; IGVH: Immunoglobulin heavy chain variable region; NK: Natural killer; BP: Biological processes.

\section{Declarations}

\section{Authors' Contributions}

Dongmei Guo, Ping Li and Zan Wang conceived and designed the experiments, analyzed the data, prepared figures and tables, authored or reviewed drafts of the paper. Banban Li and Gaofeng Bi analyzed the data, authored or reviewed drafts of the paper. Chunpu Li conceived and designed the experiments, authored or reviewed drafts of the paper, and approved the final draft.

\section{Funding}

The present study was supported by grants from the Shandong Province Medical Science and Technology Development Program (No. 2019WS206 and No. 2019WS207)

\section{Availability of data and materials}

The datasets generated during the current study are available in the GEO database(http://www.ncbi.nlm.nih.gov/geo) and the ICGC database (https://dcc.icgc.org/projects/CLLE$\mathrm{ES})$. 


\section{Ethics approval and consent to participate}

Not applicable.

\section{Consent for publication}

Not applicable.

\section{Competing interests}

The authors declare that they have no competing interests.

\section{References}

1. Rai KR, Jain P. Chronic lymphocytic leukemia (CLL)-Then and now. Am J Hematol 2016; 91: 330-340.

Karube K, Campo E. MYC alterations in diffuse large B-cell lymphomas. Semin Hematol 2015; 52: 97-106.

2. Moreno $\mathrm{C}$, Hodgson $\mathrm{K}$, Ferrer $\mathrm{G}$ et al. Autoimmune cytopenia in chronic lymphocytic leukemia: prevalence, clinical associations, and prognostic significance. Blood 2010; 116: 4771-4776.

3. Berntsson J, Nodin B, Eberhard J et al. Prognostic impact of tumour-infiltrating B cells and plasma cells in colorectal cancer. Int J Cancer 2016; 139: 1129-1139.

4. Wei Y, Lao XM, Xiao X et al. Plasma Cell Polarization to the Immunoglobulin G Phenotype in Hepatocellular Carcinomas Involves Epigenetic Alterations and Promotes Hepatoma Progression in Mice. Gastroenterology 2019; 156: 1890-1904 e1816.

5. Thorsson V, Gibbs DL, Brown SD et al. The Immune Landscape of Cancer. Immunity 2018; 48: 812-830 e814. Immunity 2018; 48: 812-830 e814.

6. Wouters MCA, Nelson BH. Prognostic Significance of Tumor-Infiltrating B Cells and Plasma Cells in Human Cancer. Clin Cancer Res 2018; 24: 6125-6135.

7. Gabrusiewicz K, Rodriguez B, Wei J et al. Glioblastoma-infiltrated innate immune cells resemble M0 macrophage phenotype. JCI Insight 2016; 1.

8. Karn T, Jiang T, Hatzis $C$ et al. Association Between Genomic Metrics and Immune Infiltration in TripleNegative Breast Cancer. JAMA Oncol 2017; 3: 1707-1711.

9. Liu X, Wu S, Yang Y et al. The prognostic landscape of tumor-infiltrating immune cell and immunomodulators in lung cancer. Biomed Pharmacother 2017; 95: 55-61.

10. Yang S, Liu T, Cheng Y et al. Immune cell infiltration as a biomarker for the diagnosis and prognosis of digestive system cancer. Cancer Sci 2019; 110: 3639-3649. 
11. Gnjatic S, Bronte V, Brunet LR et al. Identifying baseline immune-related biomarkers to predict clinical outcome of immunotherapy. J Immunother Cancer 2017; 5: 44.

12. Karube K, Campo E. MYC alterations in diffuse large B-cell lymphomas. Semin Hematol 2015; 52: 97 106.

13. Adams JM, Harris AW, Pinkert CA et al. The c-myc oncogene driven by immunoglobulin enhancers induces lymphoid malignancy in transgenic mice. Nature 1985; 318: 533-538.

14. Horn $\mathrm{H}$, Ziepert M, Becher $\mathrm{C}$ et al. MYC status in concert with BCL2 and BCL6 expression predicts outcome in diffuse large B-cell lymphoma. Blood 2013; 121: 2253-2263.

15. Kluk MJ, Chapuy B, Sinha P et al. Immunohistochemical detection of MYC-driven diffuse large B-cell lymphomas. PLoS One 2012; 7: e33813.

16. Yang $X$, Chang $Y$, Wei W. Emerging role of targeting macrophages in rheumatoid arthritis: Focus on polarization, metabolism and apoptosis. Cell Prolif 2020; 53: e12854.

17. Roberts CA, Dickinson AK, Taams LS. The Interplay Between Monocytes/Macrophages and CD4(+) T Cell Subsets in Rheumatoid Arthritis. Front Immunol 2015; 6: 571.

18. Ritchie ME, Phipson B, Wu D et al. limma powers differential expression analyses for RNA-sequencing and microarray studies. Nucleic Acids Res 2015; 43: e47.

19. Langfelder $P$, Horvath S. WGCNA: an R package for weighted correlation network analysis. BMC Bioinformatics 2008; 9: 559.

20. Zhao C. Identifying the hub gene and immune infiltration of osteoarthritis by bioinformatical methods. Clin Rheumatol 2020.

21. Li Z, Cui Y, Feng J, Guo Y. Identifying the pattern of immune related cells and genes in the peripheral blood of ischemic stroke. J Transl Med 2020; 18: 296.

22. Subramanian A, Tamayo P, Mootha VK et al. Gene set enrichment analysis: a knowledge-based approach for interpreting genome-wide expression profiles. Proc Natl Acad Sci U S A 2005; 102: 1554515550.

23. Hanzelmann S, Castelo R, Guinney J. GSVA: gene set variation analysis for microarray and RNA-seq data. BMC Bioinformatics 2013; 14: 7.

24. Newman AM, Liu CL, Green MR et al. Robust enumeration of cell subsets from tissue expression profiles. Nat Methods 2015; 12: 453-457.

25. Hallek M. Chronic lymphocytic leukemia: 2017 update on diagnosis, risk stratification, and treatment. Am J Hematol 2017; 92: 946-965. 
26. Acunzo M, Croce CM. Downregulation of miR-15a and miR-16-1 at 13q14 in Chronic Lymphocytic Leukemia. Clin Chem 2016; 62: 655-656.

27. Van Roosbroeck K, Calin GA. MicroRNAs in chronic lymphocytic leukemia: miRacle or miRage for prognosis and targeted therapies? Semin Oncol 2016; 43: 209-214.

28. Marcucci G, Maharry KS, Metzeler KH et al. Clinical role of microRNAs in cytogenetically normal acute myeloid leukemia: miR-155 upregulation independently identifies high-risk patients. J Clin Oncol 2013; 31: 2086-2093.

29. Allevato M, Bolotin E, Grossman M et al. Sequence-specific DNA binding by MYC/MAX to low-affinity non-E-box motifs. PLoS One 2017; 12: e0180147.

30. Szabo AG, Gang AO, Pedersen MO et al. Overexpression of c-myc is associated with adverse clinical features and worse overall survival in multiple myeloma. Leuk Lymphoma 2016; 57: 2526-2534.

31. Chauhan A, Paul R, Debnath M et al. Synthesis of Flourescent Binaphthyl Amines That Bind c-MYC GQuadruplex DNA and Repress c-MYC Expression.J Med Chem 2016; 59:7275-7281.

32. Hoffman B, Amanullah A, Shafarenko M, Liebermann DA. The proto-oncogene c-myc in hematopoietic development and leukemogenesis. Oncogene 2002; 21: 3414-3421.

33. Zhang J, Xiao Y, Guo Y et al. Differential requirements for c-Myc in chronic hematopoietic hyperplasia and acute hematopoietic malignancies in Pten-null mice. Leukemia 2011; 25: 1857-1868.

34. Albajar M, Gomez-Casares MT, Llorca J et al. MYC in chronic myeloid leukemia: induction of aberrant DNA synthesis and association with poor response to imatinib. Mol Cancer Res 2011; 9: 564-576.

35. Wagle $M$, Eiring $A M$, Wongchenko $M$ et al. A role for FOXO1 in BCR-ABL1-independent tyrosine kinase inhibitor resistance in chronic myeloid leukemia. Leukemia 2016; 30: 1493-1501.

36. Yun S, Vincelette ND, Segar JM et al. Comparative Effectiveness of Newer Tyrosine Kinase Inhibitors Versus Imatinib in the First-Line Treatment of Chronic-Phase Chronic Myeloid Leukemia Across Risk Groups: A Systematic Review and Meta-Analysis of Eight Randomized Trials. Clin Lymphoma Myeloma Leuk 2016; 16: e85-94.

37. Jiang Y, Saga K, Miyamoto Y, Kaneda Y. Cytoplasmic calcium increase via fusion with inactivated Sendai virus induces apoptosis in human multiple myeloma cells by downregulation of c-Myc oncogene. Oncotarget 2016; 7: 36034-36048.

38. Whiteside TL, Ferrone S. For breast cancer prognosis, immunoglobulin kappa chain surfaces to the top. Clin Cancer Res 2012; 18: 2417-2419. 
39. Nielsen JS, Sahota RA, Milne K et al. CD20+ tumor-infiltrating lymphocytes have an atypical CD27memory phenotype and together with CD8+ T cells promote favorable prognosis in ovarian cancer. Clin Cancer Res 2012; 18: 3281-3292.

40. Fregni G, Perier A, Avril MF, Caignard A. NK cells sense tumors, course of disease and treatments: Consequences for NK-based therapies. Oncoimmunology 2012; 1: 38-47.

41. Ali HR, Chlon L, Pharoah PD et al. Patterns of Immune Infiltration in Breast Cancer and Their Clinical Implications: A Gene-Expression-Based Retrospective Study. PLoS Med 2016; 13: e1002194.

42. Lad DP, Varma S, Varma $\mathrm{N}$ et al. Regulatory T-cell and T-helper 17 balance in chronic lymphocytic leukemia progression and autoimmune cytopenias. Leuk Lymphoma 2015; 56: 2424-2428.

\section{Figures}

\section{Figure 1}

DEGs screening and module-trait relationship plot. (A) Volcano plot of the differentially expressed genes in GSE31048. (B) Heatmap of the correlation between identified modules and traits in GSE31048. 
A

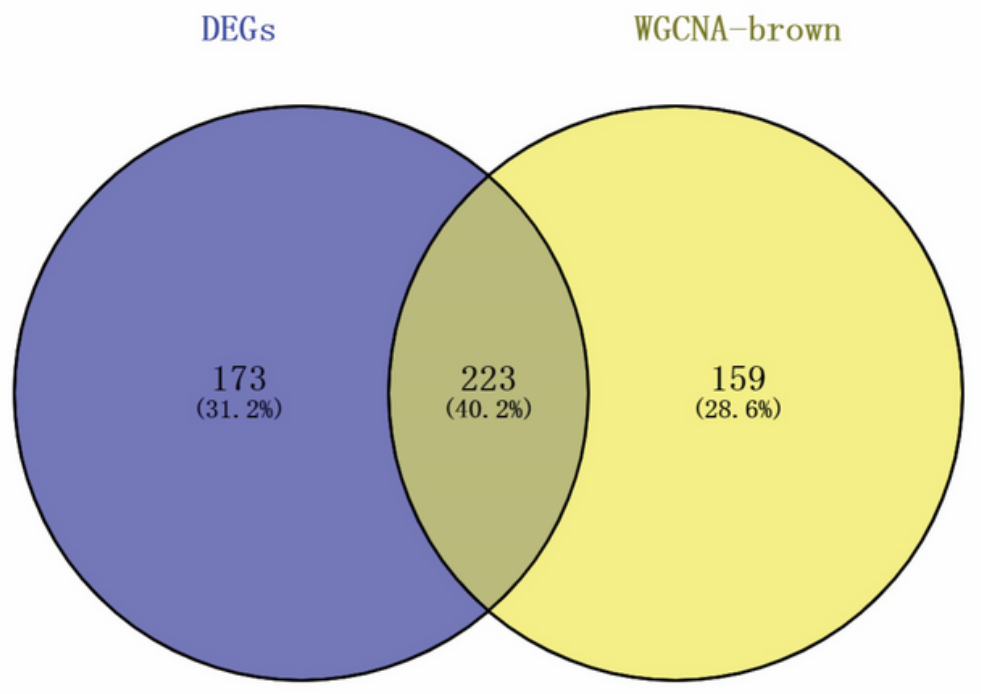

C

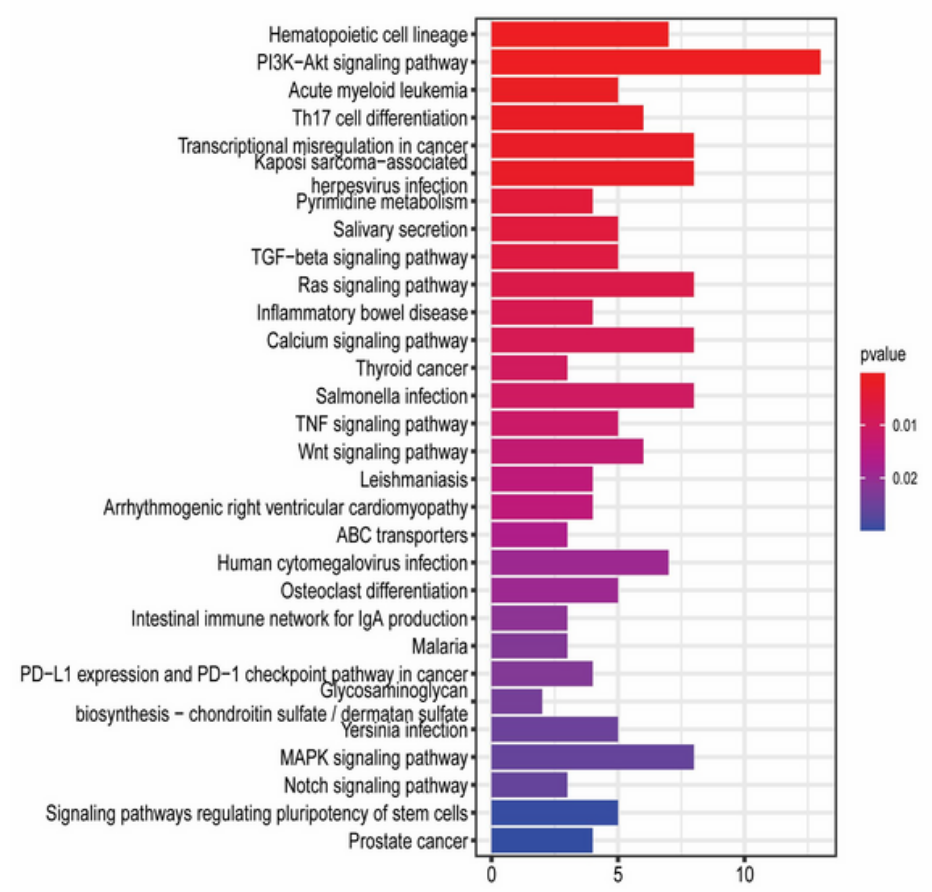

B

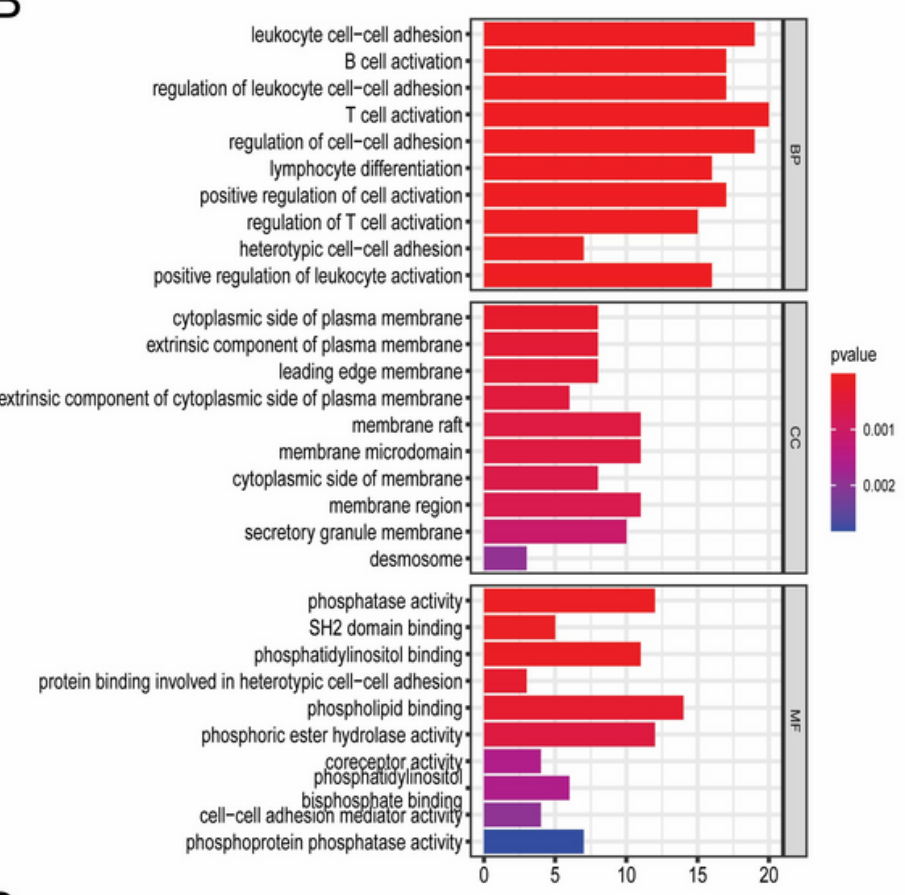

D

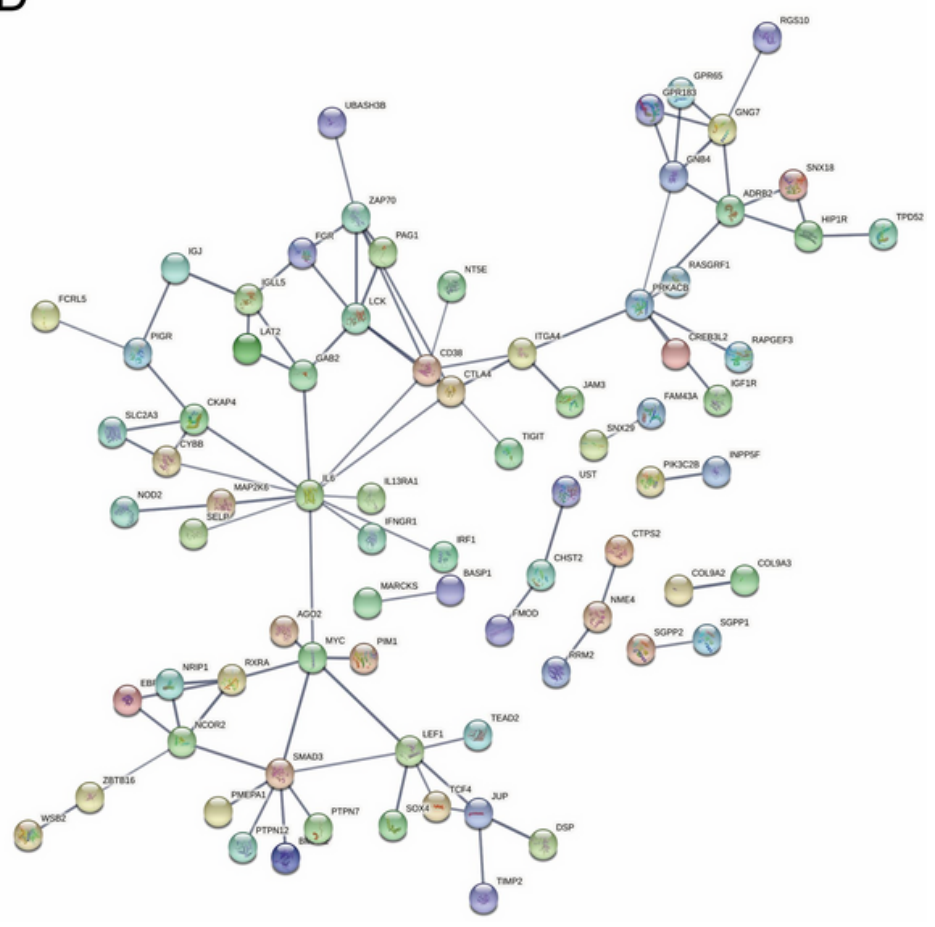

Figure 2

Identification of hub genes in CLL. (A) The Venn diagram demonstrates the 223 hub genes according to the analysis of differentially expression and WGCNA. (B) GO analysis. (C) KEGG analysis. (D) PPI network of differentially expressed protein-coding genes. 
A

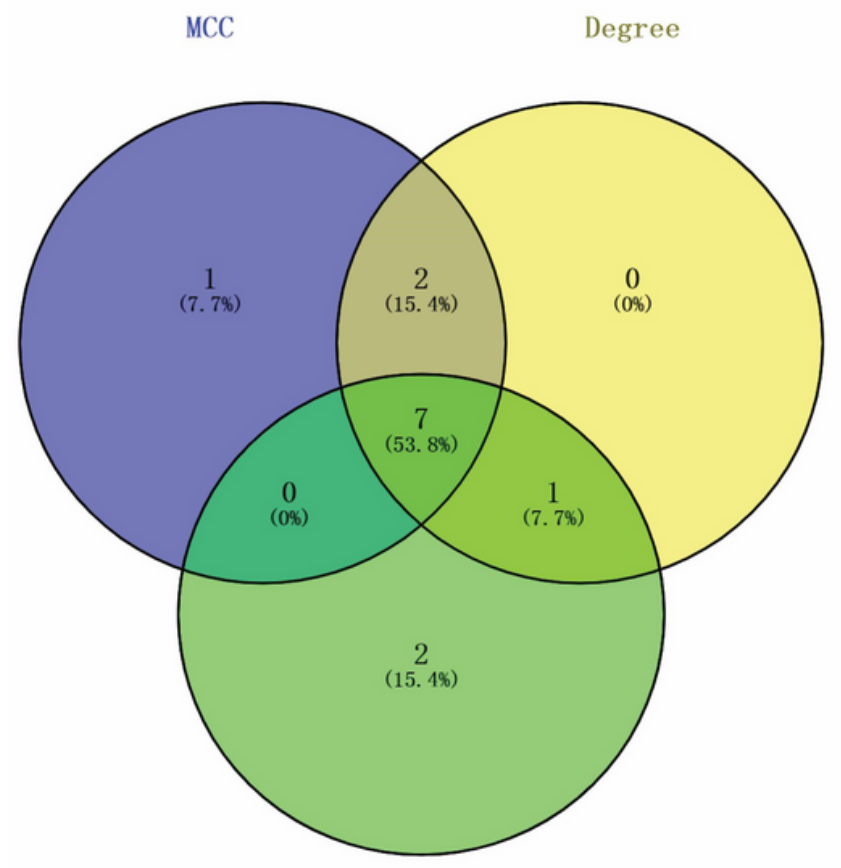

Betweenness
B

$\mathrm{MYC}->2.24(117)-<2.24(181)$

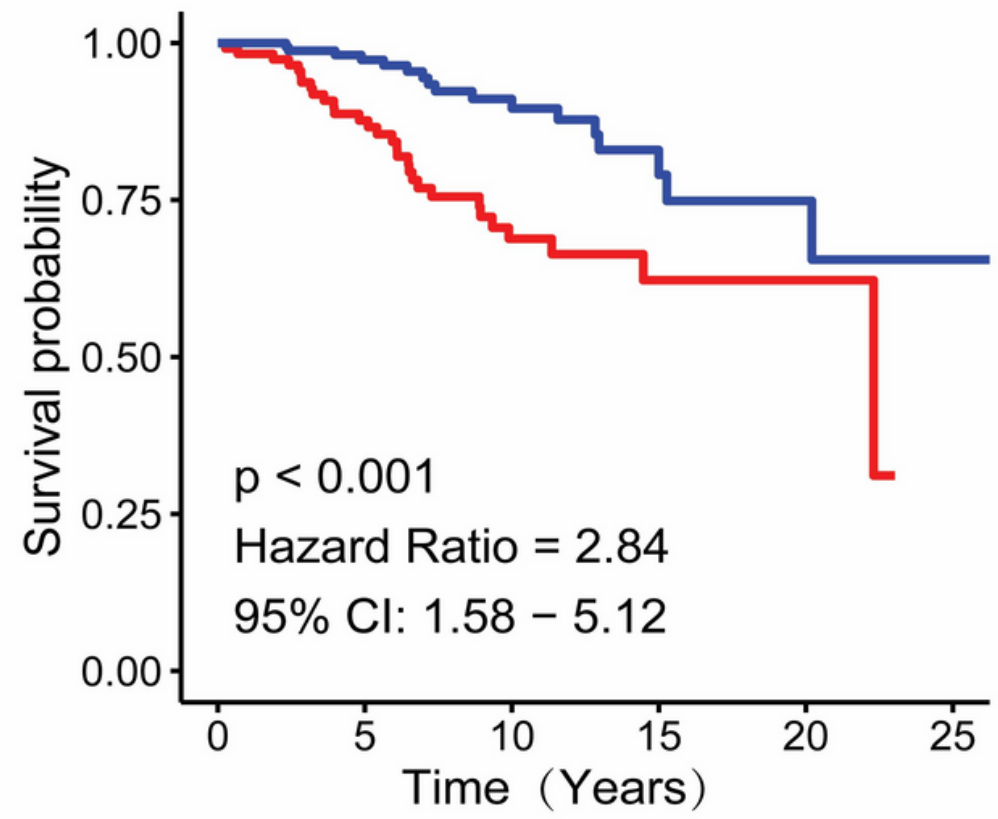

Figure 3

(A) The Venn diagram demonstrateskey genes according to three algorithms in the cytoHubba plugin. (B) Kaplan-Meier survival curve displaying the effect of high MYC expression on overall survival.

\section{Figure 4}

GSEA enrichment plot of top six signaling pathways associated with high MYC group. (A) Hypoxia. (B) MYC Targets V2. (C) TNFAsignaling via NFKB. (D) mTORC1 signaling. (E) G2Mcheckpoint. (F) P53 pathway. 


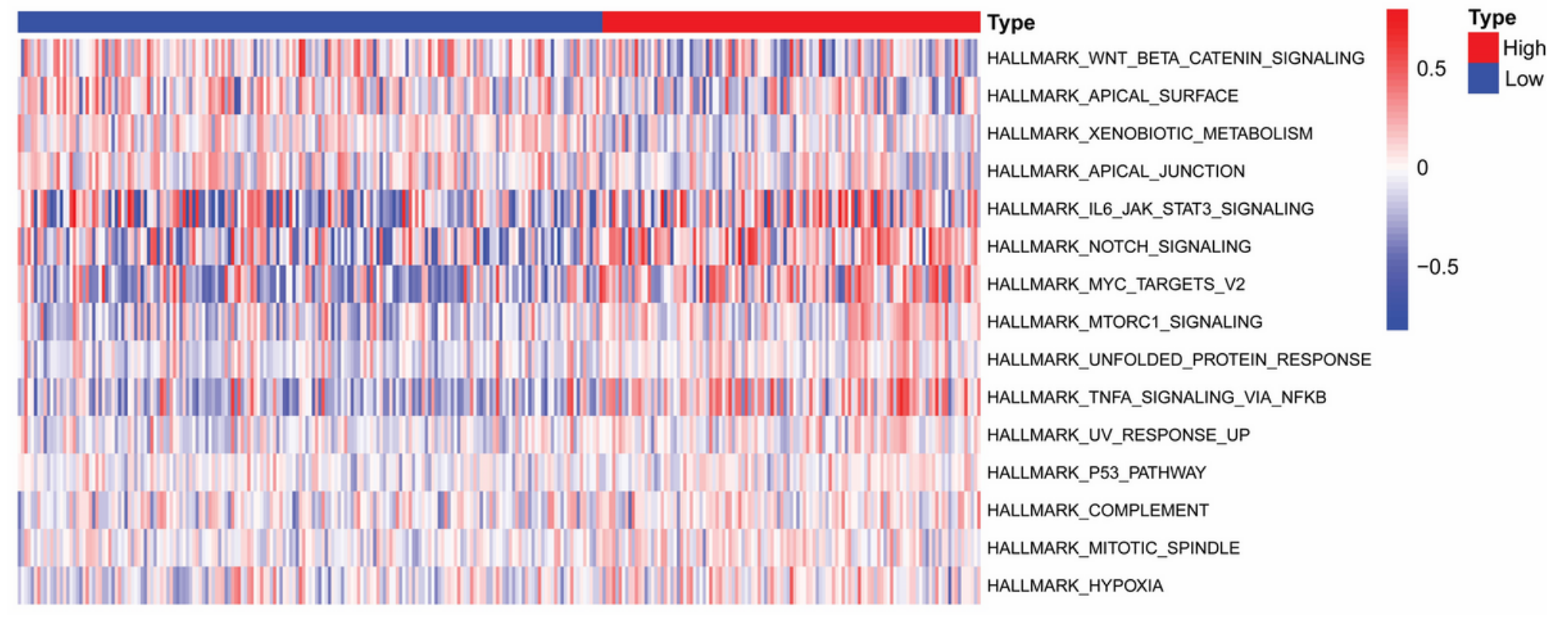

Figure 5

GSVA confirmed that the six pathways were also significantly upregulated in high MYC group.

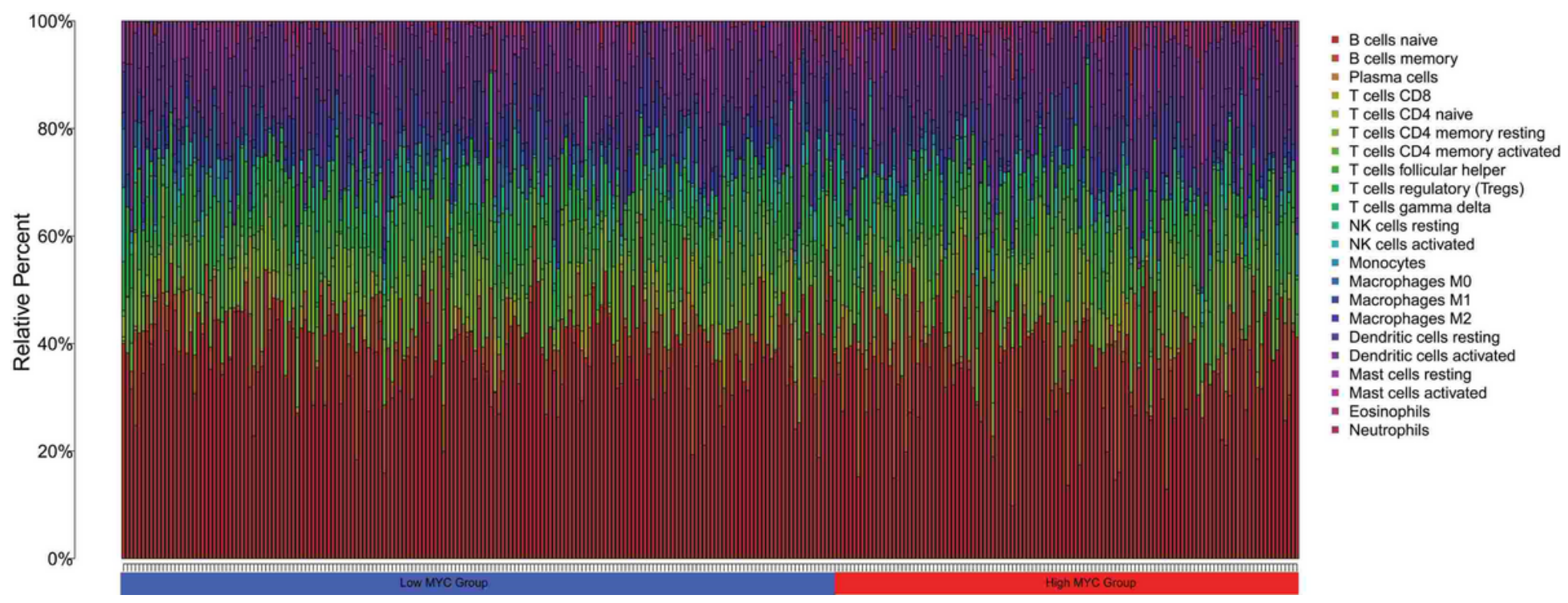

\section{Figure 6}

The association between the expression levels of MYC and immune cells. (A) The fraction of immune cell types in low and high MYC groups. (B) Violin plot of all 22 immune cells differentially infiltrated fraction (Note: The red in the figure represents high MYC group, and blue represents low MYC group). (C-E) The correlation between MYC expression level and immune cells. 
A
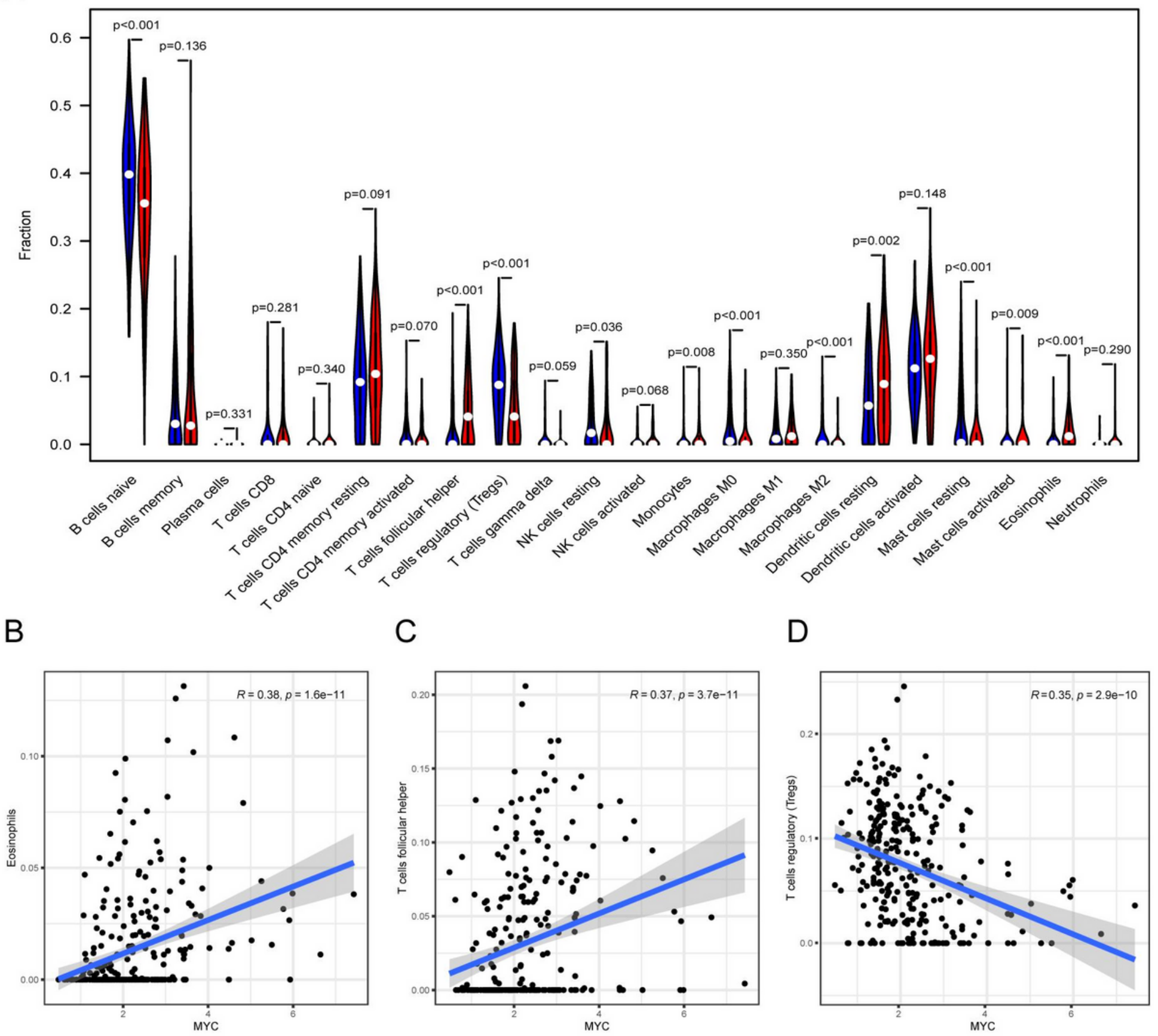

Figure 7

Legend not included with this version. 